\title{
Correction: Ten Misconceptions That Dentists Have About Treating Obstructive Sleep Apnea
}

\author{
B. Gail Demko, DMD ${ }^{1}$
}

${ }^{1}$ Sleep Apnea Dentists of New England, Newton Centre, MA

The article titled "Ten Misconceptions That Dentists Have About Treating Obstructive Sleep Apnea" [J Dent Sleep Med. 2018; 5(3):90-103] contained an incorrect description of a cited study. The error was as follows:

"In 2015 Larsen et al. evaluated records of more than 5,000 children treated at the University of Minnesota Dental School Department of Orthodontics, looking for patients who were missing bicuspids. These were case-control matched for age, sex, BMI, and presence or absence of a diagnosis of OSA confirmed by PSG. This record review determined that 267 children without missing bicuspids had received a diagnosis of OSA, and 299 children with missing bicuspids had received a diagnosis of OSA. The prevalence of OSA was therefore not significantly different between the two groups. "

The prior section should be replaced with: "In 2015, Larsen et al. reviewed the medical records of 5585 adult patients at the HealthPartners of Minnesota. Half of the patients had one bicuspid missing in each quadrant and were assumed to have had orthodontic treatment earlier in life. The subjects were case controlled, matched for age, sex, BMI, and the diagnosis of OSA confirmed by PSG. This record review determined that 267 of those without missing bicuspids had received a diagnosis of OSA and 299 subjects with missing bicuspids had received a diagnosis of OSA. The prevalence of OSA was therefore not significantly different between the two groups. ${ }^{1 "}$

The description was corrected in the manuscript, which was re-published on the JDSM website on July 24, 2018.

\section{REFERENCE} 1. Larsen AJ, Rindal DB, Hatch JP, et al. Evidence supports no relationship between obstructive sleep apnea and premolar extraction: an electronic health
records review. J Clin Sleep Med. 2015;11(12):10-15.

\section{AUTHOR CORRESPONDENCE INFORMATION}

Address correspondence to: B. Gail Demko, DMD, 6409 Prairie Dunes Drive, Grand Blanc, MI 48439, Email: bdemko@yahoo.com 